\title{
miR-215 suppresses proliferation and migration of non-small cell lung cancer cells
}

\author{
XIAOPAN CAI, DONGYU PENG, HAIFENG WEI, XINGHAI YANG, QUAN HUANG, ZAIJUN LIN, \\ WEI XU, MING QIAN, CHENG YANG, TIELONG LIU, WANGJUN YAN and JIAN ZHAO \\ Department of Bone Tumor Surgery, Changzheng Hospital, \\ The Second Military Medical University, Shanghai 200003, P.R. China
}

Received November 3, 2016; Accepted January 18, 2017

DOI: $10.3892 / \mathrm{ol} .2017 .5692$

\begin{abstract}
The expression of microRNA-215 (miR-215) in non-small cell lung cancer (NSCLC) tissues and the effects of miR-215 on the proliferation and migration of NSCLC cells were investigated. qRT-PCR was used to detect the expression of miR-215 in NSCLC tissues and paired normal tumor-adjacent lung tissues; MTT assay, transwell assay and soft-agar assay were used in vitro to evaluate the role of miR-215 on proliferation, migration and cell clonality on NSCLC cells, after transfecting miR-215 mimics to NSCLC cell line A549 or miR-215 to H1299. miR-215 was significantly decreased in NSCLC tissues compared to the paired normal tissues; Overexpression of miR-215 in A549 cells resulted in reduction of the cell proliferation, migration and cell clonality, while downregulation of miR-215 in H1299 cells could promote cell proliferation, migration and clonality. In conclusion, miR-215 was downregulated in NSCLC tissues and may play a key role in the development of NSCLC.
\end{abstract}

\section{Introduction}

Lung cancer is one of the major cancer-related deaths in the world. As a result of tumor heterogeneity, late diagnosis and high recurrence, the 5-year survival rate of lung cancer is only $16 \%$ (1). Non-small cell lung cancer (NSCLC) is the most common lung cancer, accounting for $\sim 85 \%$ of the total (2). In recent years, high-throughput sequencing has provided effective tools to deeply investigate changes of genetics and epigenetics in the development of lung cancer (3). Targeting the molecular changes in lung cancer cannot only help to find effective treatments, but also play key role in risk assessment,

Correspondence to: Dr Jian Zhao, Department of Bone Tumor Surgery, Changzheng Hospital, The Second Military Medical University, 415 Fengyang Road, Shanghai 200003, P.R. China

E-mail: drzhaojian@189.cn; pk8b99@163.com

Key words: non-small cell lung cancer, migration, microRNA, lung cancer, miR-215 early diagnosis, more accurate staging and typing, recurrence and prognosis assessment in lung cancer (4).

MicroRNA (miRNA or miR) has aroused great attention in the field of cancer biology since it can regulate various cell activities $(5,6)$. As a kind of none-coding RNA, abnormal expression of miRNA is frequent in many cancers, including lung cancer (7), and it can play a role of tumor suppressor or tumor promoter in different cancers (8). miR-215 is highly expressed in normal nerve tissues, which can promote the development of the nervous system and maintain its normal physiological functions (9). As reported, miR-215 is highly related to cell proliferation, differentiation, apoptosis, migration and invasion in various cancers, including colon, gastric and breast cancer (10-12). Nevertheless, little is known about its function in lung cancer. In this study, we first detected the expression of miR-215 in NSCLC tissues. Afterwards, NSCLC cell lines A549 and H1299 were chosen to evaluate the role of miR-215 in the development and progression of NSCLC.

\section{Materials and methods}

Tissue specimen preparation. A total of 30 cases of NSCLC patients were selected from Changzheng Hospital (Shanghai, China) in July 2013. Sample collection was performed after receiving approval from the Institutional Ethics Review Committee of Changzheng Hospital. No patient had undergone chemotherapy or radiation before surgery. Lung cancer tissues and paired normal tumor-adjacent lung tissues (normal appearance lung tissues and $\geq 5 \mathrm{~cm}$ away from cancer tissues) were selected. This study was approved by the ethics committee of Changzheng Hospital. Signed written informed consents were obtained from all participants before the study.

Cells and major regents. Human NSCLC cell lines A549 and H1299 were purchased from American Type Culture Collection (ATCC). miR-215 mimics, miR-215 inhibitor and negative control miRNA were purchased from RiboBio Co., Ltd. (Guangzhou, China). The quantitative PCR (qPCR) reactions and TRIzol reagent were purchased from Takara Bio, Inc. (Otsu, Japan). RPMI-1640 and Opti-MEM were purchased from Gibco Life Technologies (Carlsbad, CA, USA). Trypsin-EDTA solution was purchased from Hyclone (Logan, 
UT, USA). Lipofectamine ${ }^{\circledR} 2000$ was purchased Invitrogen Life Technologies (Carlsbad, CA, USA).

Cell culture. A549 and $\mathrm{H} 1299$ cell lines were grown in RPMI-1640, supplemented with $10 \% \mathrm{FBS}$ at $37^{\circ} \mathrm{C}$ and $5 \% \mathrm{CO}_{2}$. Cells were divided into three groups: Negative control group, miR-215 overexpression group and miR-215 downregulated group. Lipofectamine ${ }^{\circledR} 2000$ was used to transfect negative control miRNA, miR-215 mimics and miR-215 inhibitor to different cells according to manufacturer's protocol.

$q P C R$. Detection of miR-215 in tissues and cells. After grinding, total RNA was extracted from tissues using TRIzol reagent and reversely transcribed using the PrimeScript ${ }^{\mathrm{TM}} \mathrm{RT}$ Reagent kit. qPCR was subsequently performed according to the manufacturer's instructions. The expression levels were normalized against the internal reference gene U6, and the relative expression levels were displayed using the $2^{-\Delta \Delta \mathrm{Cq}}$ method. The miRNA sequence-specific RT-PCR primers for miR-215 and endogenous control RNU6B were purchased from Ambion Life Technologies (Carlsbad, CA, USA). In addition, total RNA of cells were extracted after transfecting for $24 \mathrm{~h}$, and miR-215 was detected according to above-mentioned methods.

Western blotting. Cell lysates were extracted with the extraction reagent. Equal amounts of total proteins $(20 \mu \mathrm{g})$ were separated by $10 \%$ SDS-PAGE and transferred onto PVDF membrane. After blocking for nonspecific binding, the membranes were incubated with anti-PCNA (1:1,000 dilution; Cell Signaling Technology, Inc., Danvers, MA, USA), or GAPDH (1:5,000 dilution; Bioworld Technology, Inc., St. Louis Park, MN, USA) overnight at $4^{\circ} \mathrm{C}$, followed by HRP-conjugated secondary antibodies for $1 \mathrm{~h}$ at $25^{\circ} \mathrm{C}$. After washing three times in TBST, protein bands were visualized using chemiluminescence detection.

MTT assay. A total of 1,000 transfected cells in $200 \mu 1$ of medium were incubated in 96-well culture plates. Cell proliferation was assayed using a 3-(4,5-dimethylthiazol-2-yl)-2, 5-diphenyltetrazolium bromide (MTT) kit and a Synergy H4 Hybrid Reader. Briefly, the culture medium was removed after $1,2,3$ and 4 days, and $0.5 \mathrm{mg} / \mathrm{ml}$ MTT in $200 \mu \mathrm{l}$ of medium was added to each well and incubated for $4 \mathrm{~h}$. The cells were then treated with $150 \mu$ l DMSO for $10 \mathrm{~min}$, and the optical density measured at $540 \mathrm{~nm}$. Each experiment was repeated in triplicate.

Transwell assay. Cell migration was performed by the tranwell assay. Briefly, $5 \times 10^{4}$ cells in serum-free RPMI-1640 were seeded on a membrane (8.0- $\mu \mathrm{m}$ pore size) inserted in the wells of a 24-well plate. RPMI-1640 containing $10 \%$ FBS was added to the lower chamber of each well. After $24 \mathrm{~h}$, cells in the upper chamber were removed by cotton swab and the cells that had reached the underside of the membrane were fixed and stained with crystal violet (1\% in methyl alcohol) for $30 \mathrm{~min}$. After washed with PBS 3 times, the cells located on the underside of the filter (5 fields/filter) were counted.

Soft-agar assay. Plates $(60 \mathrm{~mm})$ were covered with a layer of $0.66 \%$ agar in medium supplemented with $20 \%$ FBS. Cells

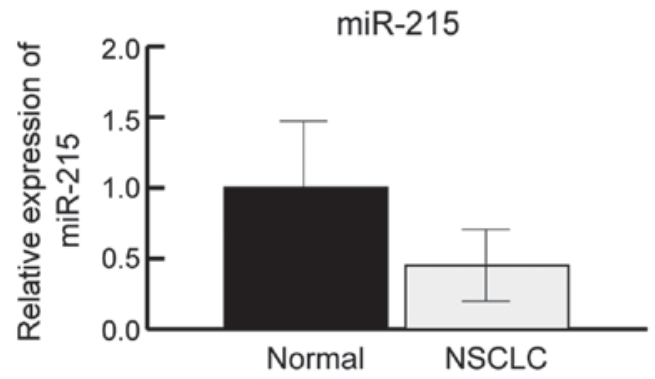

Figure 1. miR-215 expression in normal and non-small cell lung cancer (NSCLC) tissues.

$\left(1 \times 10^{4}\right)$ were seeded on the top of agar layer and cultured in RPMI-1640 supplemented with 10\% FBS with $0.3 \%$ agarose. The cells were incubated with $5 \% \mathrm{CO}_{2}$ at $37^{\circ} \mathrm{C}$. After 2-3 weeks, the number of cell colonies were counted under a microscope and cell colonies were photographed in 100x view. Colonies of $>50$ cells were counted. The experiment was repeated 3 times independently, for each cell line. Data are presented as the mean \pm standard deviation $(\mathrm{SD})$.

Statistical analysis. SPSS 19.0 software (IBM SPSS, Armonk, NY, USA) was used for statistical analysis. All quantitative data were expressed as mean $\pm \mathrm{SD}$. Comparison between groups was done using one-way ANOVA test followed by post hoc test (least significant difference). Percentage (\%) was used to express the enumeration data and Chi-square test was used for data analysis. $\mathrm{P}<0.05$ was considered to indicate a statistically significant difference.

\section{Results}

Detection of miR-215 in NSCLC tissues. qPCR was used to detect the expression of miR-215 in lung cancer tissues and paired normal tumor-adjacent lung tissues of 30 NSCLC cases. Results showed that miR-215 expression was significantly decreased in lung cancer tissues compared with paired normal tumor-adjacent lung tissues (Fig. 1).

Detection of miR-215 expression in transfected lung cancer cells with qPCR. qPCR was performed to detect inner expression of miR-215 in NSCLC cell lines A549 and H1299, results showed that expression of miR-215 was higher in H1299 than that in A549 (Fig. 2A). MiR-215 mimics was transfected to A549 cells, while miR-215 inhibitor was transfected to H1299 cells. After $24 \mathrm{~h}, \mathrm{qPCR}$ was used to detect the expression of miR-215. As shown in Fig. 2B, miR-215 expression was significantly elevated after miR-215 mimics transfection, while miR-215 expression was significantly decreased with miR-215 inhibitor transfection (Fig. 2C).

The effect of miR-215 on NSCLC cell proliferation. To detect the effect of miR-215 on NSCLC cell proliferation, A549 cells were transfected with miR-215 mimics, and H1299 cells were transfected with miR-215 inhibitor. Afterwards, MTT assay was used to detect the effect of miR-215 on NSCLC cell proliferation. Results showed that overexpression of miR-215 inhibited the proliferation of A549 cells, and decreased the 
A

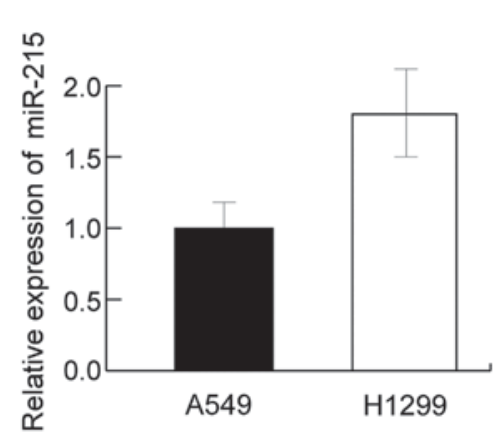

B

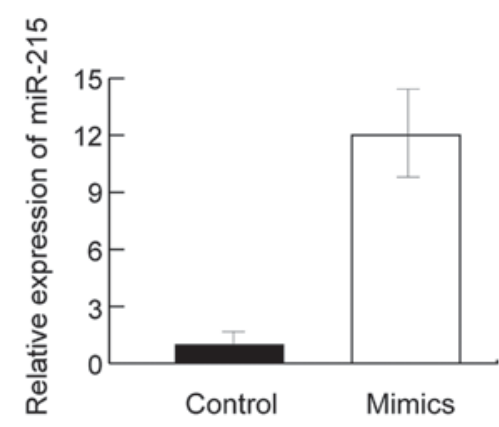

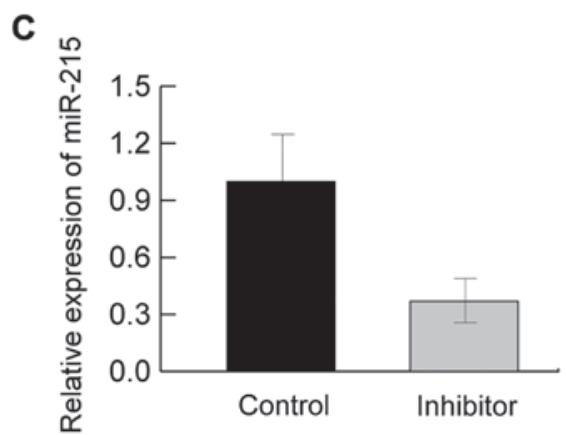

Figure 2. miR-215 expression in transfected lung cancer cells: (A) miR-215 expression in A549 and H1299 cell lines; (B) miR-215 expression was significantly elevated after transfection with miR-215 mimics; (C) miR-215 expression was significantly decreased with miR-215 inhibitor transfection.

expression of PCNA. Nevertheless, knocking down miR-215 could promote the proliferation of $\mathrm{H} 1299$ cells, and increased the expression of PCNA. The differences were statistically significant (Fig. 3).

The effect of miR-215 on NSCLC cell migration. To detect the effect of miR-215 on NSCLC cell migration, A549 cells were transfected with miR-215 mimics, and H1299 cells were transfected with miR-215 inhibitor. Afterwards, transwell assay showed that overexpression of miR-215 inhibited the migration of A549 cells, while knocking down of miR-215 promoted the proliferation of H1299 cells (Fig. 4). These results suggested that miR-215 was able to regulate migration of NSCLC cells.

The effect of miR-215 on anchorage-independent growth activity of NSCLC cell. To detect the effect of miR-215 on anchorage-independent growth activity of NSCLC cells, A549 cells were transfected with miR-215 mimics, and H1299 cells were transfected with miR-215 inhibitor. Results showed that, as compared to control group, overexpression of miR-215 inhibited cell clonality, while knocking down of miR-215 promoted cell clonality (Fig. 5). These results suggested that miR-215 could affect the anchorage-independent growth activity of NSCLC cells.

\section{Discussion}

At present, malignant tumor is the first leading cause of death in the world, while lung cancer is the leading cause of cancer-related death. The main target and hotspot of lung cancer research is to pursue early diagnosis and proper selection of drugs and adjuvant treatment according to pathogenesis of lung cancer, thus improving the survival rate and quality

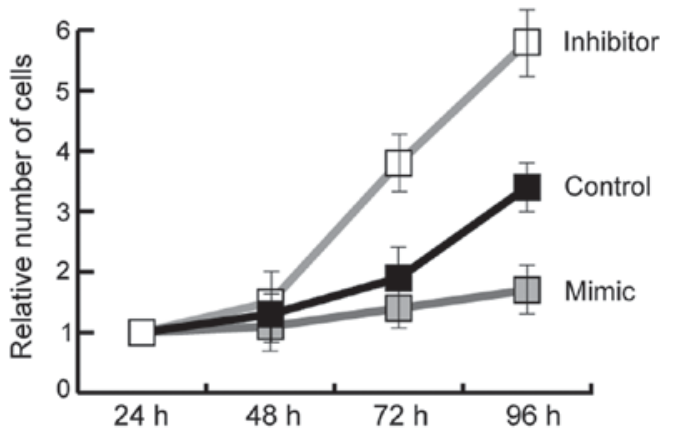

Figure 3. miR-215 inhibits proliferation of non-small cell lung cancer cells.

of life of cancer patients (13-15). Moreover, except for deep investigations into lung cancer, little is known about the accurate mechanisms of pathogenesis and metastasis of lung cancer. miRNAs and other small non-coding RNAs have been shown to be important regulators of gene expression, and the fundamental function of miRNAs is to regulate their targets by direct cleavage of the mRNA or by inhibition of protein synthesis. Abundant evidence suggests that miRNA play a vital role in tumor development and progression (16). In NSCLC, many miRNAs have been reported to regulate cancer cell proliferation and migration by targeting various oncogene or tumor suppressor gene, such as miR-99b, miR-101 and miR-192, whose target, respectively, was FGFR3, EZH2 and RB1 (17-19). Although the mechanisms related to how miRNAs promote or inhibit cancer are high spots in tumor investigation, there are still many functions of miRNA that remain to be explored.

miR-215 is highly expressed in the nervous system, and plays an important role in development and normal physiological functions maintaining the nervous system (9). Li et al 

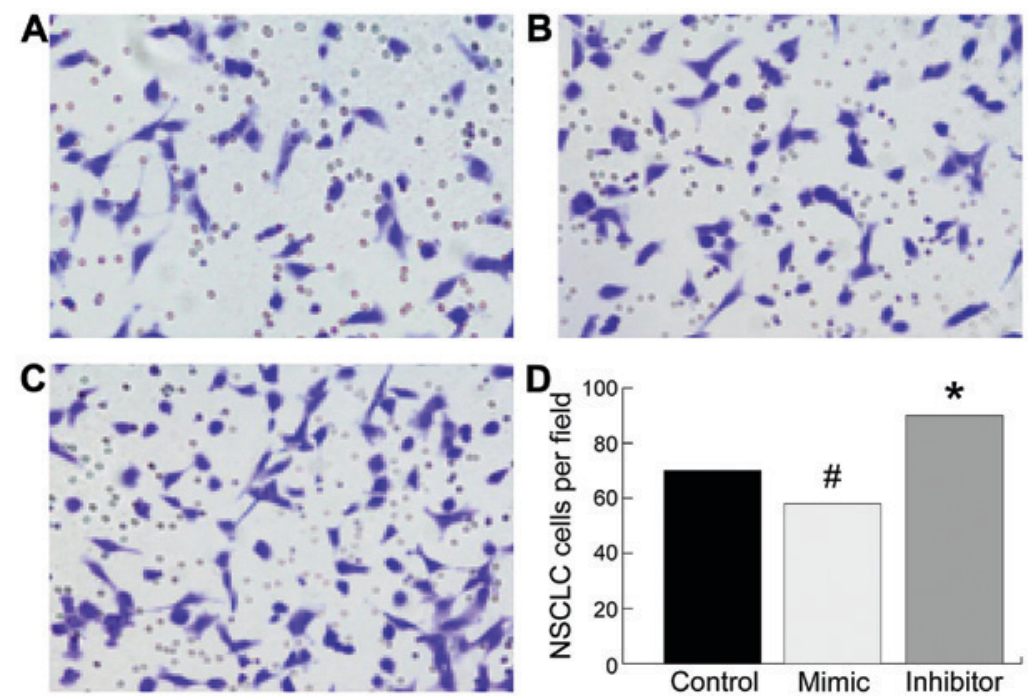

Figure 4. miR-215 attenuates migration of non-small cell lung cancer (NSCLC) cells. (A) Control group; (B) cells transfected with miR-215 mimics; (C) cells transfected with miR-215 inhibitors; (D) overexpression of miR-215 inhibit the migration while knocking down of miR-215 have the opposite effects. ${ }^{\#} \mathrm{P}<0.05$ compared with control; " $\mathrm{P}<0.05$ compared with control.
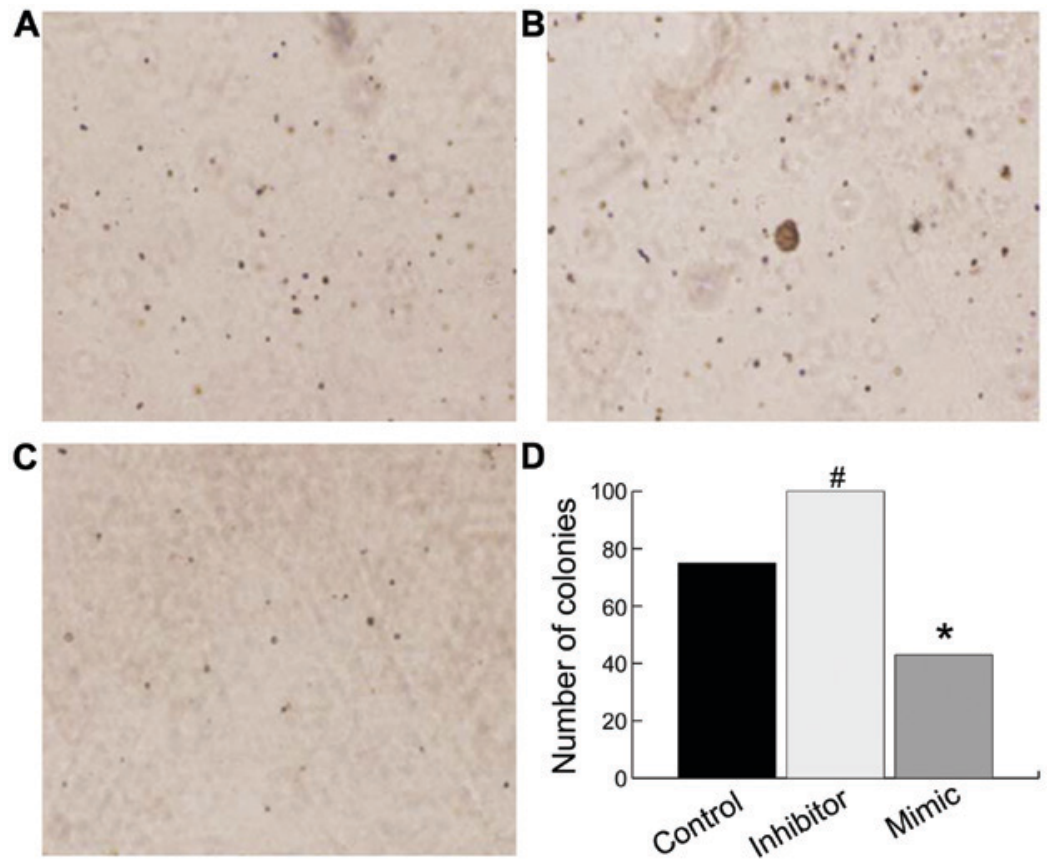

Figure 5. The effects of miR-215 on cell clonality in non-small cell lung cancer cells. (A) Control group; (B) cells transfected with miR-215 inhibitors; (C) cells transfected with miR-215 mimics; (D) overexpression miR-215 inhibits cell clonality, while knocking down miR-215 promotes cell clonality. ${ }^{*} \mathrm{P}<0.05$ compared with control; ${ }^{\mathrm{P}}<0.05$ compared with control.

found that miR-215 can promote malignant progression of gastric cancer by targeting RUNX1 (11). Zhou et al (12) reported that miR-215 was associated with the clinical outcome in breast cancer patients. miR-215 also have very important functions in other tumors. In ovarian cancer, miR-215 functions as a tumor suppressor in epithelial ovarian cancer through regulation of the $\mathrm{X}$-chromosome-linked inhibitor of apoptosis (20). In osteosarcoma cells, miR-215, through the suppression of DTL expression, can induce decreased cell proliferation by causing G2-arrest, thereby leading to an increase in chemo-resistance to MTX and TDX (21). Above all, evidence suggests that miR-215 may act as a tumor promoter or tumor suppressor in different cell environments.

Little is known about miR-215 in NSCLC cells. As Ye et al reported, the low expression of miR-215 in NSCLC was due to the loss of heterozygosity, thus leading to high expression of its target gene EGFR (19). This suggested that miR-215 is not only related to the development and progression of NSCLC, but can also provide new strategies for lung cancer treatment. There is no report yet on whether miR-215 could influence the biological function of NSCLC cells. We found that miR-215 was lowly expressed in NSCLC tissues, and overexpression of miR-215 could inhibit lung cancer cell proliferation and 
clonality. This result conforms to what Tong et al (22) reported, as they found that miR-215 could inhibit proliferation of glioma cells by targeting CTNNBIP1/ $\beta$-catenin pathway. In addition, we found that miR-215 inhibited NSCLC cell migration. As reported in ovarian cancer and colon cancer, miR-215 was a negative regulator of cell migration $(20,21)$. In conclusion, all the evidence suggests that abnormal expression of miR-215 is closely related to malignant tumor including NSCLC.

Through negative regulating target genes, miRNA plays a key role in cell various activities. Seeking target gene and related mechanisms of miR-215 in NSCLC is helpful to deeply investigate the mechanisms of tumor inhibition of miR-215, thus providing new strategies of prevention, early diagnosis and treatment for NSCLC.

\section{References}

1. de Groot P and Munden RF: Lung cancer epidemiology, risk factors, and prevention. Radiol Clin North Am 50: 863-876, 2012 .

2. Molina JR, Yang P, Cassivi SD, Schild SE and Adjei AA: Non-small cell lung cancer: Epidemiology, risk factors, treatment, and survivorship. Mayo Clin Proc 83: 584-594, 2008.

3. Didkowska J, Wojciechowska U, Mańczuk M and Łobaszewski J: Lung cancer epidemiology: Contemporary and future challenges worldwide. Ann Transl Med 4: 150, 2016.

4. Morales-Espinosa D, García-Román S, Karachaliou N and Rosell R: Pharmacogenomics in the treatment of lung cancer: An update. Pharmacogenomics 16: 1751-1760, 2015.

5. Yanaihara N, Caplen N, Bowman E, Seike M, Kumamoto K, Yi M, Stephens RM, Okamoto A, Yokota J, Tanaka T, et al: Unique microRNA molecular profiles in lung cancer diagnosis and prognosis. Cancer Cell 9: 189-198, 2006.

6. Yu SL, Chen HY, Chang GC, Chen CY, Chen HW, Singh S, Cheng CL, Yu CJ, Lee YC, Chen HS, et al: MicroRNA signature predicts survival and relapse in lung cancer. Cancer Cell 13: 48-57, 2008.

7. Rabinowits G, Gerçel-Taylor C, Day JM, Taylor DD and Kloecker GH: Exosomal microRNA: A diagnostic marker for lung cancer. Clin Lung Cancer 10: 42-46, 2009.

8. Yongchun Z, Linwei T, Xicai W, Lianhua Y, Guangqiang Z, Ming Y, Guanjian L, Yujie L and Yunchao H: MicroRNA-195 inhibits non-small cell lung cancer cell proliferation, migration and invasion by targeting MYB. Cancer Lett 347: 65-74, 2014.

9. White NM, Khella HW, Grigull J, Adzovic S, Youssef YM Honey RJ, Stewart R, Pace KT, Bjarnason GA, Jewett MA, et al: miRNA profiling in metastatic renal cell carcinoma reveals a tumour-suppressor effect for miR-215. Br J Cancer 105 : 1741-1749, 2011.
10. Karaayvaz M, Pal T, Song B, Zhang C, Georgakopoulos P, Mehmood S, Burke S, Shroyer K and Ju J: Prognostic significance of miR-215 in colon cancer. Clin Colorectal Cancer 10: 340-347, 2011.

11. Li N, Zhang QY, Zou JL, Li ZW, Tian TT, Dong B, Liu XJ, Ge S, Zhu Y, Gao J, et al: miR-215 promotes malignant progression of gastric cancer by targeting RUNX1. Oncotarget 7: 4817-4828, 2016.

12. Zhou SW, Su BB, Zhou Y, Feng YQ, Guo Y, Wang YX, Qi P and $\mathrm{Xu} \mathrm{S}$ : Aberrant miR-215 expression is associated with clinical outcome in breast cancer patients. Med Oncol 31: 259, 2014.

13. Hirsch FR, Franklin WA, Gazdar AF and Bunn PA Jr: Early detection of lung cancer: Clinical perspectives of recent advances in biology and radiology. Clin Cancer Res 7: 5-22, 2001.

14. Olezac AS, Papanikolaou I, Bengrine-Lefevre L and Chouaid C: Choriocarcinoma with pulmonary metastasis: Diagnosis and treatment. Rev Mal Respir 26: 769-772, 2009 (In French).

15. Sollini M, Farioli D, Froio A, Chella A, Asti M, Boni R, Grassi E, Roncali M, Versari A and Erba PA: Brief report on the use of radiolabeled somatostatin analogs for the diagnosis and treatment of metastatic small-cell lung cancer patients. J Thorac Oncol 8: 1095-1101, 2013.

16. Liu XH, Liu ZL, Sun M, Liu J, Wang ZX and De W: The long non-coding RNA HOTAIR indicates a poor prognosis and promotes metastasis in non-small cell lung cancer. BMC Cancer 13: 464, 2013.

17. Kang J, Lee SY, Lee SY, Kim YJ, Park JY, Kwon SJ, Na MJ, Lee EJ, Jeon HS and Son JW: microRNA-99b acts as a tumor suppressor in non-small cell lung cancer by directly targeting fibroblast growth factor receptor 3. Exp Ther Med 3: 149-153, 2012.

18. Chen S, Wang H, Ng WL, Curran WJ and Wang Y: Radiosensitizing effects of ectopic miR-101 on non-small-cell lung cancer cells depend on the endogenous miR-101 level. Int J Radiat Oncol Biol Phys 81: 1524-1529, 2011.

19. Ye M, Zhang J, Zhang J, Miao Q, Yao L and Zhang J: Curcumin promotes apoptosis by activating the p53-miR-192-5p/215-XIAP pathway in non-small cell lung cancer. Cancer Lett 357: 196-205, 2015.

20. Ge G, Zhang W, Niu L, Yan Y, Ren Y and Zou Y: miR-215 functions as a tumor suppressor in epithelial ovarian cancer through regulation of the X-chromosome-linked inhibitor of apoptosis. Oncol Rep 35: 1816-1822, 2016.

21. Song B, Wang Y, Titmus MA, Botchkina G, Formentini A, Kornmann M and Ju J: Molecular mechanism of chemoresistance by miR-215 in osteosarcoma and colon cancer cells. Mol Cancer 9: 96, 2010.

22. Tong YQ, Liu B, Zheng HY, Gu J, Liu H, Li F, Tan BH, Hartman M, Song C and Li Y: MiR-215, an activator of the CTNNBIP1/ $\beta$-catenin pathway, is a marker of poor prognosis in human glioma. Oncotarget 6: 25024-25033, 2015. 